\title{
The Effect of an Integrated Information System Adoption for the Health Promotion in Patients with Laparoscopic Colon Resection
}

\author{
Seong-Ran Lee* \\ Department of Medical Information, Kongju National University \\ lsr2626@naver.com
}

\begin{abstract}
The purpose of this study is to analyze the effect of an integrated information system adoption for the health promotion in patients with laparoscopic colon resection. The subjects of this paper were patients who had visited a general hospital which located in the area of metropolitan from July 8 to September 10, 2013. The total of 152 subjects were divided into experimental group 76 and control group 76. As a result, first, the present research showed the mean score of green tea drinking after intervention increased significantly than subjects $(t=-5.28, p=0,000)$ before the intervention Second, the present research showed that the rate of the health improvement behavior of colon cancer patients can be increased to 75.4-83.2\% by the intervention. Therefore, the paper will contribute to the improvement in the quality of life through application of an integrated information system to solve problems from patients with laparoscopic colon resection.
\end{abstract}

Keywords: Integrated information program, Effect, Health promotion, Patients, Laparoscopic colon resection

\section{Introduction}

Colon cancer is one of the major malignancies in western countries and its incidence is rapidly increasing in other countries including Japan [1]. Moreover, the incidence of colon cancer in Korea has also increased dramatically over the past few decades.

According to the National Cancer Registry, the age-standardized incidence rates increased from 29.1 to 46.5 per 100,000 for men and from 19.2 to 26.3 per 100,000 for women between 2000 and 2010. The overall incidence of colon cancer increased by $9.0 \%$ annually in men and $5.3 \%$ in women from 2000 to 2010 , while the incidence rates of the most common cancers, such as stomach and liver cancers, decreased during the same period. Epidemiologic studies have suggested that higher intakes of red and processed meat, a lack of physical activity, obesity, and alcohol drinking are risk factors for colon cancer. During the past few decades, significant socio-economic changes have taken place in Korea with respect to the economy, industrial structure, dietary habits, physical activity, and prevalence of obesity. Changes in dietary habits and lifestyle-related factors are believed to be the reasons for the increase in the incidence of colon cancer in Korea [1-3].

Although a higher rate was observed in males than in females, these rates were fourand eightfold higher in African Americans and Immigrant Koreans, when compared with their white counterparts in both sexes.

* This work was supported by the research grant of the Kongju National University in 2013 
Despite a substantial reduction of colon cancer incidence in the United States and other Western, it remains the most frequent malignancy in native and immigrant Koreans. The high rate of colon cancer in immigrant Koreans compared with African Americans and white populations residing in Illinois indicates either a drastically disproportionate undercount of immigrant Koreans in the 1990 census or a profound genetic-environmental interaction $[1,4,5]$.

The median age of colon cancer patients is over 70 years. Recently, the effect of treatment locally advanced colon cancer has been reported that it could be different by age. Therefore, this study was conducted to evaluate health practice factors including psychiatric factors related to health improvement in patients after laparoscopic colon resection. Risk factors for local recurrence and indication for preoperative radiation therapy have not been well evaluated for patients undergoing laparoscopic colon cancer operation [6, 7]. From 2003 to 2009, 497 T3 colon cancer patients with tumor located within $12 \mathrm{~cm}$ from the anal verge who had undergone laparoscopic surgery without preoperative radiation therapy by eight experienced laparoscopic surgeons in four Asian countries were reviewed retrospectively for the incidence of local recurrence and related factors. The median follow-up was 29.0 months (range, 6.0 to 92.3), and 31 cases of local recurrence were observed during the follow-up period ( 6 anastomosis site, 6 perineum, 17 pelvic wall, and 2 unclassified). The estimated local recurrence rates at 24 and 60 months were 5.42 and $9.41 \%$, respectively. Patient's gender, tumor location, lymph node metastasis, and tumor perforation were independent factors for local recurrence by multivariate analysis. The local recurrence rate was comparable to previous studies using conventional open surgery with preoperative chemoradiation, except for a subgroup of male patients with the tumor located within $7 \mathrm{~cm}$ from the anal verge $[8,9]$. The indication for preoperative radiation therapy would be different from those who will undergo conventional open surgery, and further evaluation of the benefits of preoperative radiation therapy is required for those with low risk tumor.

Recent increase of colorectal cancer has been attributed to high intake of fat and protein. However, there are inconsistent results between meat intake and the incidence of colorectal cancer. Recently the frequency of the colon cancer in the laparoscopic colon resection is increasing with the increased use of health check-up laparoscopy. Laparoscopic treatment is becoming increasing used as an alternative to traditional surgical resection, but there are few reports on the efficacy, safety and long-term prognosis of laparoscopic treatment for colon cancer $[10,11]$.

In order to solve the problem, we should look for the practical plans. There were few studies to deal with information application of predictive model for health promotion in patients with laparoscopic colon surgery until present in Korea. This study is to examine the relationship between information system application and health promotion behavior of patients who had surgery for colon cancer.

Thus, we need to develop a health management program for laparoscopic surgery patients. An integrated information system is asked rapidly for more efficiency and demand response in recent. As the utilization of efficient health management has been receiving considerable attention in recent years, the information system requirement is increased. Specially, the reliability information system is more important for implementing the health performation for patients with laparoscopic colon resection. This paper describes an integrated information system that simulates the reliability indices in information system considering colon resection patients developed successfully in this paper. We verified the establishment of integrated information system based on intervention applications of integrated information system. This system will provide an integrated health information and be a very useful tool to support the decision making process of effective change adaptation policies. Moreover, the system will 
contribute to the role of the intervention to solve problems stemming from an increasing colon cancer patients and related patients problems. In addition, this study designed to develop the long-term health intervention program and ultimately to analyze the intervention effect through its application.

Therefore, the purpose of this paper is the effect of an integrated information system adoption for the health promotion in patients with laparoscopic colon resection. That is, this study is to conduct a comparative verification on the development of information system program suitable for colon cancer patients to improve their physical and psychological health. Because the study has verified the effect of an information program to boost colon cancer patients health through a scientific measurement method, it is expected that the study will help with an improvement in their quality of life through an information

\section{Materials and Methods}

\subsection{Construction of Information System}

This study is to construct effectively database system by making use of intervention effect method. The first step of the program development is to determine the necessity of the participants. The second step is to identify the functional elements of successful models and gather the information about this. In the third step, program formation which will be reflected in effectively database system is designed as apart of information gathering and synthesis. The four step is the stage of information system analysis and design. In the five step, the stage of operation, where preliminary program applied in the field has been implemented. The final step is to assess the effect of personal information and build up the procedure [Figure 1].

\subsection{Materials}

From July 8 to September 10, 2013, we screened patients with colon cancer who received laparoscoipic resection in $\mathrm{K}$ general hospital. The total of 152 subjects were divided into experimental group 76 and control group 76. The program was held 6 times per week for 8 weeks.

The instrument for testing patients undergoing depression with hysterectomy consists of a total of 22 items. The first items for general characteristics are consisted 8 items of age, gender, marital status, economic status, educational level, occupation, family history, other disease. The second items for health practice rate for a depression prevention are consisted 13 items of physical factors(cholesterol, hypertension, blood sugar control), psychological factors(depression, anxiety, perceived stress, sleep disorder), dietary factors(vegetable intake, meat intake, green tea drinking), social factors(passive smoking, drinking alcohol, daily living activity).

\subsection{Methods}

All statistical analyses were carried out using SAS package program (version 10.0 for Windows) for descriptive analysis, $\chi^{2}$-test, and t-test.

General characteristics of study subjects were measured by percentage and number. $\chi^{2}$-test was used to determine the general characteristics of the subjects including education level. The pairwise t-test was done to compare the differences in the scores between the pretest and posttest of the intervention effect designed to develop information system and conducted in two groups. On the other hand, the estimate of the four factors had been implemented to find out whether there were possible effects of the health promoting behavior program of a new information system by analyzing the results of the tests. 


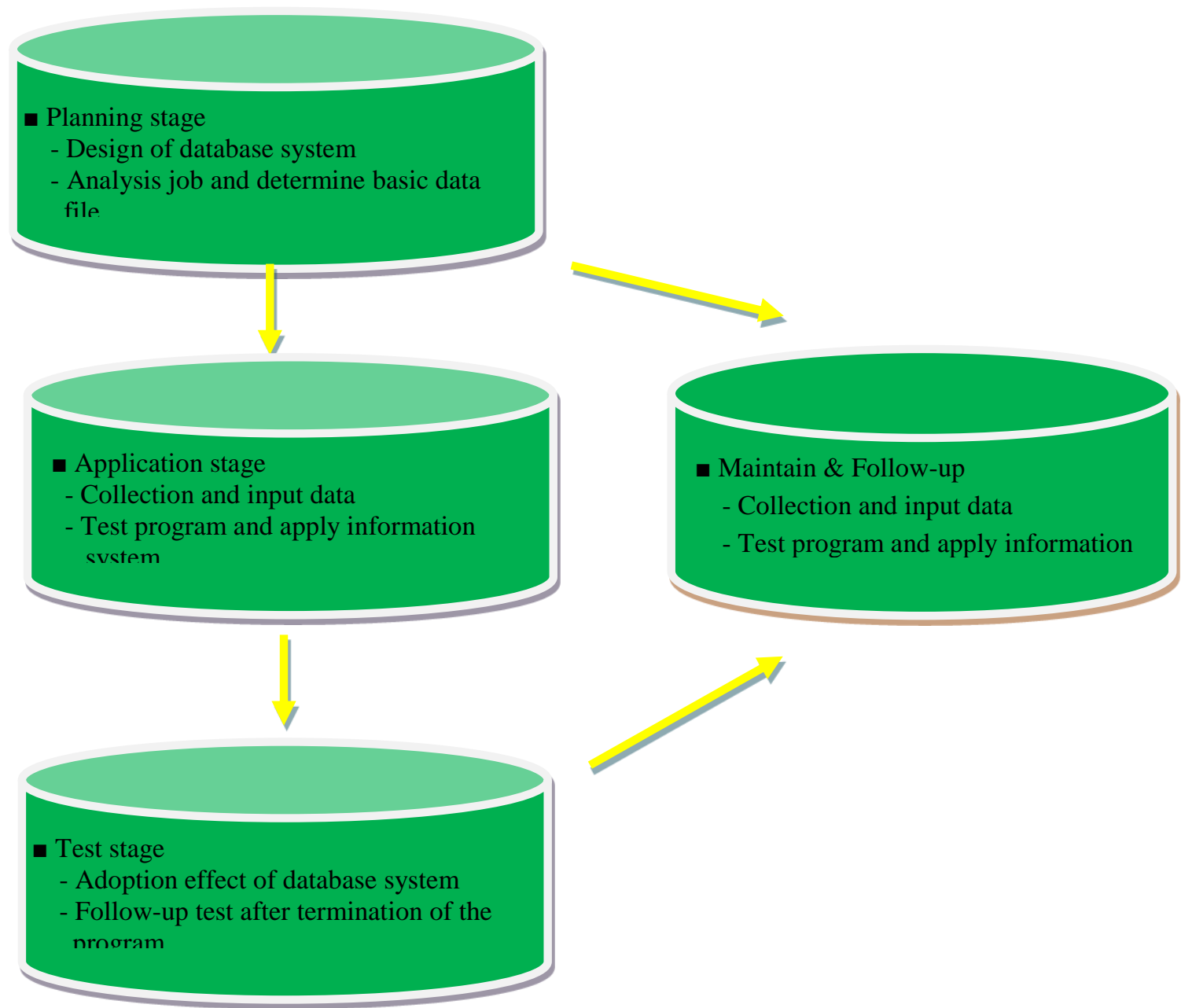

Figure 1. Predictive Model for Health Promoting Behavior of Colon Cancer Patients

\section{Result}

\subsection{General Characteristics of Study Subjects}

Table 1 presents general characteristics of study subjects. The response rate $(46.1 \%)$ of experimental groups was higher than the response rate(40.8\%) of control groups at male according to gender of subjects. On the other hand, for subjects with a family history, the response rate $(75.0 \%)$ of experimental groups was statistically significantly higher than the response rate $(38.2 \%)$ of control groups $\left(\mathrm{X}^{2}=3.86, \mathrm{p}=.007\right)$. The response rate $(59.2 \%)$ of experimental group with other disease was statistically significantly higher than the response rate $(31.6 \%)$ of the control group $\left(\mathrm{X}^{2}=7.24, \mathrm{p}=.015\right)$ according to other diseases of subjects. 
Table 1. General Characteristics of Study Subjects

\begin{tabular}{|c|c|c|c|c|}
\hline Variables & $\begin{array}{l}\text { Experimental } \\
\text { Group(N=76) }\end{array}$ & $\begin{array}{l}\text { Control } \\
\text { group }(\mathrm{N}=76)\end{array}$ & $X^{2}$ & $\mathrm{p}$ \\
\hline \multicolumn{5}{|l|}{ Age } \\
\hline$\leq 39$ & $9(11.8)$ & $12(15.8)$ & 12.85 & .062 \\
\hline $40-49$ & $26(34.2)$ & $21(27.6)$ & & \\
\hline $50-59$ & $22(28.9)$ & $19(25.0)$ & & \\
\hline$\geq 60$ & $19(25.0)$ & $24(31.6)$ & & \\
\hline \multicolumn{5}{|l|}{ Gender } \\
\hline Male & $35(46.1)$ & $31(40.8)$ & 4.70 & .295 \\
\hline Female & $41(53.9)$ & $45(59.2)$ & & \\
\hline \multicolumn{5}{|l|}{ Marital status } \\
\hline Single & $20(26.3)$ & $17(22.4)$ & 6.95 & .072 \\
\hline Married & $56(73.7)$ & $59(77.6)$ & & \\
\hline \multicolumn{5}{|c|}{ Economic status/millions } \\
\hline$<2$ & $19(25.0)$ & $23(30.3)$ & 13.58 & .169 \\
\hline $2-2.99$ & $23(30.3)$ & $25(32.9)$ & & \\
\hline $3-4.99$ & $20(26.3)$ & $17(22.4)$ & & \\
\hline$\geq 5$ & $14(18.4)$ & $11(14.5)$ & & \\
\hline \multicolumn{5}{|l|}{ Educational } \\
\hline$\leq$ middle school & $8(10.5)$ & $14(18.4)$ & 10.46 & .461 \\
\hline High school & $32(42.1)$ & $32(42.1)$ & & \\
\hline$\geq$ University & $36(47.4)$ & $30(39.5)$ & & \\
\hline \multicolumn{5}{|l|}{ Occupation } \\
\hline Yes & $47(61.8)$ & $44(57.9)$ & 5.91 & .198 \\
\hline No & $29(38.2)$ & $32(42.1)$ & & \\
\hline \multicolumn{5}{|l|}{ Family history } \\
\hline Yes & $57(75.0)$ & $29(38.2)$ & 3.86 & .007 \\
\hline No & $19(25.0)$ & $47(61.8)$ & & \\
\hline \multicolumn{5}{|l|}{ Other disease } \\
\hline Yes & $45(59.2)$ & $24(31.6)$ & 7.24 & .015 \\
\hline No & $31(40.8)$ & $52(68.4)$ & & \\
\hline Total & $76(100.0)$ & $76(100.0)$ & & \\
\hline
\end{tabular}

A chi-square test (also chi-squared or X) is any statistical hypothesis test in which the sampling distribution of the test statistic is a chi-square distribution when the null hypothesis is true, or any in which this is asymptotically true, meaning that the sampling distribution (if the null hypothesis is true) can be made to approximate a chi-square distribution as closely as desired by making the sample size large enough [1].

$$
\begin{aligned}
& \mathrm{X}^{2}=\frac{\Sigma(0-E)^{2}}{\mathrm{E}} \\
& \mathrm{df}=(\mathrm{C}-1)(\mathrm{R}-1) \\
& \mathrm{C}=\text { category number of column } \\
& \mathrm{R}=\text { category number of row }
\end{aligned}
$$




\subsection{Comparison of Health Promotion Behavior by Intervention}

Table 2 presents the comparison of health promotion behavior of before and after intervention of a new information system. Below table notes, according to the health practice scores after intervention, the mean score of green tea drinking after intervention displayed a significant increase than subjects $(\mathrm{t}=5.28, \mathrm{p}=0.000)$ before the intervention. And then behavioral change of meat intake after intervention was significantly lower compared to before the intervention $(\mathrm{t}=7.05, \mathrm{p}=0.000)$.

Table 2. The Comparison of Health Promotion Behavior by Intervention

\begin{tabular}{lcccc}
\hline \multirow{2}{*}{ Variables } & Before & After & & \\
\cline { 2 - 3 } Physical factors & Mean \pm S.D. & Mean \pm S.D. & $\mathrm{T}$ & \\
Exercise & & & & \\
Body weight & $57.81 \pm 0.36$ & $76.35 \pm 0.54$ & -2.63 & .007 \\
Cholesterol & $71.53 \pm 1.64$ & $50.81 \pm 1.59$ & 8.19 & .024 \\
Hypertension & $76.81 \pm 0.51$ & $69.24 \pm 0.65$ & 1.52 & .753 \\
Blood sugar & $82.94 \pm 1.63$ & $70.38 \pm 0.47$ & 7.36 & .392 \\
Psychological factors & $74.53 \pm 1.64$ & $62.79 \pm 1.85$ & 5.47 & .165 \\
Depression & & & & \\
Anxiety & $47.28 \pm 0.73$ & $45.72 \pm 0.21$ & 1.62 & .703 \\
Perceived stress & $65.07 \pm 0.44$ & $47.36 \pm 1.72$ & 4.29 & .019 \\
Sleep disorder & $63.45 \pm 0.26$ & $52.51 \pm 0.68$ & 2.83 & .062 \\
Dietary factors & $71.53 \pm 1.64$ & $50.81 \pm 1.54$ & 5.12 & .038 \\
Vegetable intake & & & & \\
Meat intake & $63.94 \pm 0.82$ & $83.19 \pm 0.42$ & -3.94 & .002 \\
Green tea drinking & $78.21 \pm 0.54$ & $42.57 \pm 0.81$ & 7.05 & .000 \\
Social factors & $31.82 \pm 1.71$ & $63.94 \pm 1.46$ & 5.28 & .000 \\
Passive smoking & & & &. \\
Drinking alcohol & $51.68 \pm 0.52$ & $32.39 \pm 0.62$ & 0.29 & .019 \\
Daily living activity & $72.95 \pm 0.18$ & $65.70 \pm 0.28$ & 3.67 & .042 \\
\hline
\end{tabular}

Suppose that the $\mathrm{n}$ measurements $\mathrm{X}_{1}, \mathrm{X}_{2} \ldots, \mathrm{X} n$ make up a sample [2]. Then the sample standard deviation, denoted s, is defined to be in which $\mathrm{X}$ is the mean of the measurements [3].

Mean $=$ Sum of $\mathrm{X}$ values $/ \mathrm{N}$ (Number of values)

$$
\begin{aligned}
s & =\sqrt{\frac{\left(X_{1}-\bar{X}\right)^{2}+\left(X_{2}-\bar{X}\right)^{2}+\cdots+(X n-\bar{X})^{2}}{n-1}} \\
& =\sqrt{\frac{\sum_{i=1}^{n}\left(X_{i}-\bar{X}\right)^{2}}{n-1}}
\end{aligned}
$$

$\mathrm{T}$-value $=($ Difference between group means $) /($ variability of groups $)$

$=\frac{\bar{X}_{T}-\bar{X}_{C}}{S E\left(\bar{X}_{T}-\overline{X c}\right)}$ 
The paired t-test is actually a test that the differences between the two observations is 0 . So, if $D$ represents the difference between observations, the hypotheses are $: p$-value associated with it is low $(p<0.05)$, there is evidence to reject the null hypothesis. Thus, this would have evidence that there is a difference in means across the paired observations [4].

Ho : $D=0$ (the difference between the two observations is 0 )

Ha : $D O \neq 0$ (the difference is not 0 )

\subsection{The Effect of A New Information System According to Physical Factors}

Figure 2 presents the effect of a new information system according to physical factors. The physical factors after one week by intervention were significantly lower in the experimental groups compared to control groups $(\mathrm{p}<.05)$. With regard to physical factors, it increased gradually over the period in the experimental groups compared with the control groups after one week. It increased steadily in the experimental groups until four weeks. However, there was a slight decrease in the experimental groups during the period after four weeks.

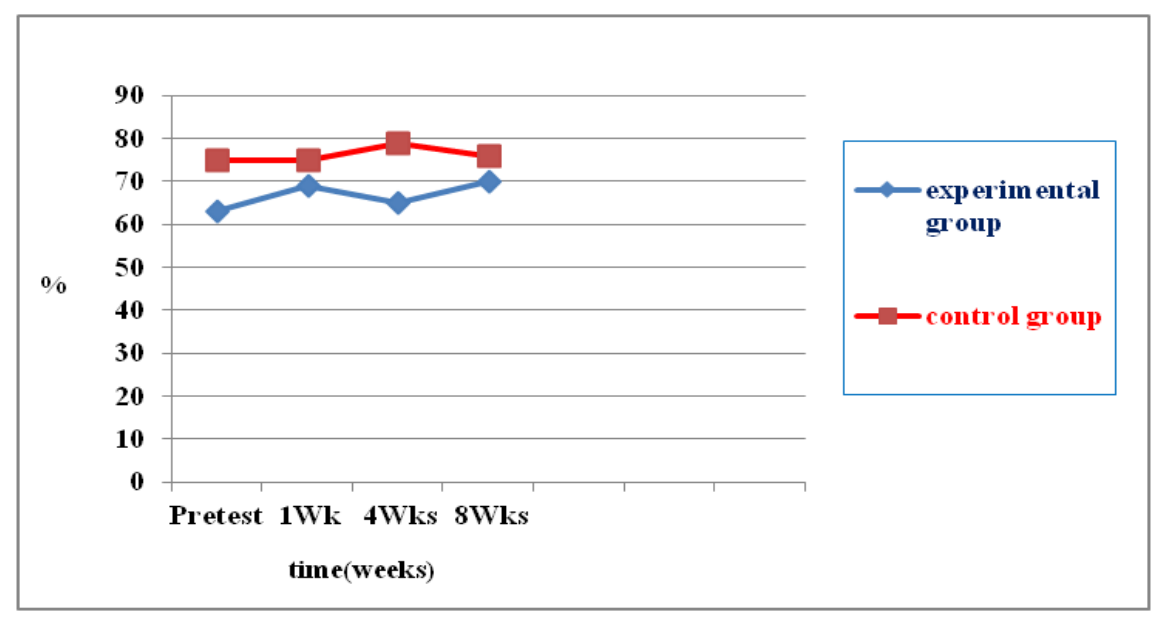

Figure 2. Physical Factors of An Integrated Information System

\subsection{The Effect of A New Information System According to Psychological Factors}

Figure 3 presents the effect of a new information system according to psychological factors. The physical factors after one week by intervention were significantly lower in the experimental groups compared to control groups $(\mathrm{p}<.05)$. With regard to psychological factors, it decreased gradually over the period in the experimental groups compared with the control groups after one week. Moreover, it decreased steadily in the experimental groups. However, there was a slight increase in the control groups during the period after four weeks. 


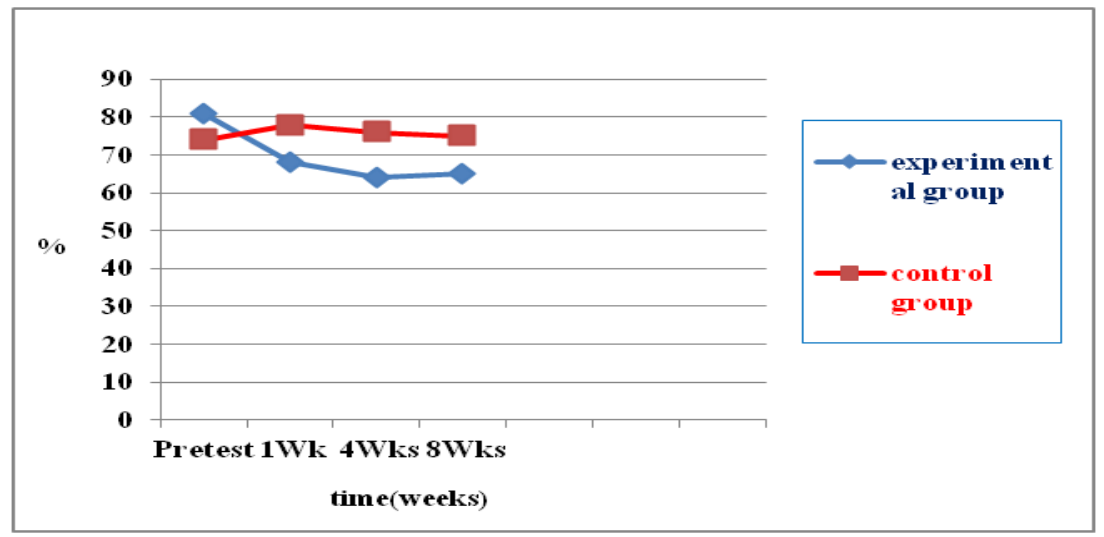

Figure 3. Psychological Factors of An Integrated Information System

\subsection{The Effect of A New Information System According to Dietary Factors}

Figure 4 presents the effect of a new information system according to dietary factors. The physical factors after four weeks by intervention were significantly lower in the experimental groups compared to control groups( $\mathrm{p}<.05)$. With regard to dietary factors, it decreased gradually over the period in the experimental groups compared with the control groups after one week. It decreased steadily in the experimental groups but there was a slight increase in the control groups during the period after four weeks.

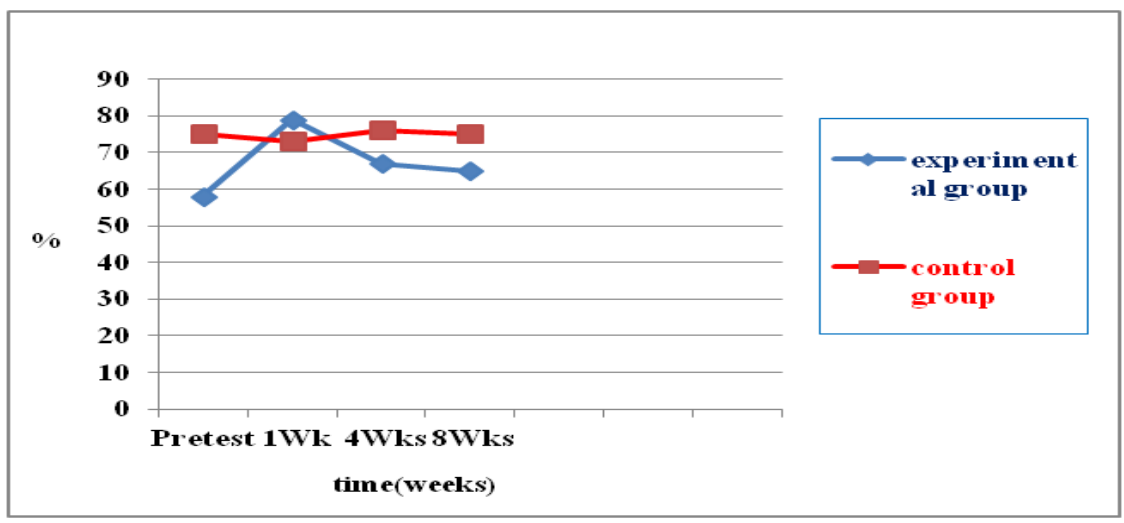

Figure 4. Dietary Factors of An Integrated Information System

\subsection{The Effect of A New Information System According to Social Factors}

Figure 5 presents the effect of a new information system according to social factors. The physical factors after eight weeks by intervention were significantly lower in the experimental groups compared to control groups $(\mathrm{p}<.05)$. With regard to social factors, it increased gradually over the period in the experimental groups compared with the control groups after one week. However, it decreased steadily in the experimental groups but there was a slight increased in the control groups during the period after four weeks. Comparing social factors of the both groups, the average rate of the passive smoking for the control group had a slight increase but the experimental group had more serious diminution after four weeks. 


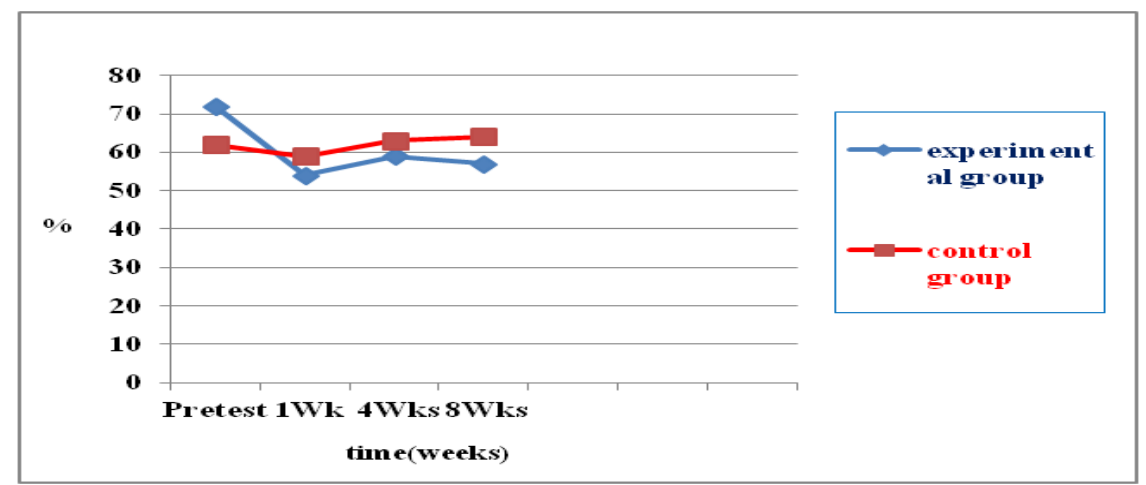

Figure 5. Social Factors of an Integrated Information System

\section{Discussion}

The purpose of this research is to develop a new information system-based self-care promoting program for self-management of colon patients with laparoscopic surgery and identify the effects of this program on patients' self-care behavior, nutritional status, and quality of life. This paper also shows the feasibility of the system through some tests.

This material is based on the research of finding out any difference in the scores between the pretest and posttest of the intervention effect designed to develop information system and conducted in two groups. As a result, green tea intake may be the tumor suppressive effects of dietary green tea extracts as a modulator on cisplatin in an established colon cancer model. The finding is similar to previous researches $[12,13]$.

As the result shows, both the experimental and the control group measured body weights and depression had slight decreases. However, experimental group's body fat decreased while the control group's it was increased. Through the result of this study, we found that there is significant result in the experimental group. Also the there no change on abdominal fat in the experimental group but there was actual increase in the control group. Comparing cholesterol control of patients with laparoscopic colon resection based on the intervention whether information apply or not, there was no noticeable difference discovery between circumstances of cholesterol control before and after information application, and there was no reciprocal action occurred by above situations. These findings suggest that the application of a new information system may be effective to reduce the obesity and increase the vegetable intake of subjects.

The data suggested that meditation had positive impact on health-promoting behaviors of patients with laparoscopic colon resection. Information intervention with an emphasis on meditation is needed to increase health promoting behaviors and, in turn to improve quality of life among colon cancer patients who have undergone surgical treatment. There is significant result in the exercise group. The finding is similar to previous researches $[14,15]$. Therefore, the information-based exercise for colon resection patients would be a novel intervention to increase shoulder, circumstance of wrist, and elbow area.

The study was found that experimental group in meditation had a tendency toward gradually less variation in depression in experimental group than control group over the timings of measurement. This finding indicates that mediation program is effective in relieving from depression. This finding also shows that exercise is effective in improving general health and vitality of colon resection patients. With regard to measurement of stress over measurement timings, it was also found that exercise group had a tendency toward 
gradually higher social role performance, self-confidence, general health and vitality over the timings of measurement, but had a tendency toward gradually lower vegetable intake over measurement timings. This finding indicates that exercise is effective in alleviating colon cancer patients from stress.

In short, intervention information conducted to improve physical and psychological factors of the subjects to socially expected standards and help patients to decrease their cholesterol and triglyceride level with efficiency. Thus, this paper revealed that the implemented systematic model of a new information system showed significant positive effects on the quality of life in patients with colon resection. In addition, it is concluded that integrated health information systems are effective in relieving patients with laparoscopic colon resection from depression over the physical, psychological, and social changes which they had experienced.

Therefore, the results of this study suggest that the information-based health promoting program as proposed in the research for self-management of patients with colon resection would be effective in increasing patients' degree of self-efficacy, self-care behavior, nutritional status and quality of life.

\section{Conclusion}

The purpose of this study is to analyze the effect of an integrated information system adoption for the health promotion in patients with laparoscopic colon resection.

Subjects of this study were patients who visited general surgery in K general hospital in Metropolitan from July 8 to September 10, 2013. The total of 152 subjects were divided into experimental group 76 and control group 76. General characteristics of study subjects were measured by percentage and number. The pairwise t-test was done to compare the differences in the scores between the pretest and posttest of the intervention effect designed to develop information system and conducted in two groups. On the other hand, the estimate of the four factors had been implemented to find out whether there were possible effects of the health promoting behavior program of a new information system by analyzing the results of the tests. The results of this paper were as follows.

First, for subjects with a family history, the response rate(75.0\%) of experimental groups was statistically significantly higher than the response rate $(38.2 \%)$ of control groups $\left(\mathrm{X}^{2}=3.86\right.$, $\mathrm{p}=.007)$. The response rate $(59.2 \%)$ of experimental group with other disease was statistically significantly higher than the response $\operatorname{rate}(31.6 \%)$ of the control $\operatorname{group}\left(\mathrm{X}^{2}=7.24, \mathrm{p}=.015\right)$ according to other diseases of subjects.

Second, according to the health practice scores after intervention, the mean score of green tea drinking after intervention displayed a significant increase than subjects $(t=5.28, p=0.000)$ before the intervention. And then behavioral change of meat intake after intervention was significantly lower compared to before the intervention $(\mathrm{t}=7.05, \mathrm{p}=0.000)$.

Third, comparing social factors of the both groups, the average rate of the passive smoking for the control group had a slight increase but the experimental group had more serious diminution after four weeks.

Fourth, the present research showed that the rate of the health improvement behavior of patients with laparoscopic colon resection can be increased to $75.4-83.2 \%$ by the intervention.

Therefore, it is concluded that this program is effective in relieving patients with laparoscopic resection from stress over physical, psychological and social changes which they had experienced in personal life, family and local community in the face of their years of life, and is also an alternative program fit for improvement of their general health. Moreover, it is important to extend the coverage of this exercise program into wider population, so that more 
people have possibility and opportunity to enjoy better and healthier life. In addition, a new information system-based health promoting program can be utilized as an effective intervention for self-management useful for any other cancer patients, for clinical practice and for home care. Therefore, information-based health promoting program can be utilized as an effective nursing intervention for self-management of colon cancer patients with resection. This program can also be found useful for any cancer patients.

\section{References}

[1] Statistics Korea, "Annual Report on the Cause of Death Statistics", (2013).

[2] A. M. Fitzgerald-Smith, P. Shrivastava, and M. J. Hershman, "The Role of the Nurse in Colorectal Cancer Follow-up", Hospital Medicine, vol. 64, no. 6, (2003), pp. 344-347.

[3] S. C. Galloway, and J. E. Graydon, "Uncertainty Symptom Distress and Information Needs After Surgery for Cancer of the Colon", Cancer Nursing, vol. 19, no. 2, (2006), pp. 112-117.

[4] W. G. Ouchi, B. Markets and W. Royce, "Software Project Management", A Unified Framework, AddisonWesley, (2001).

[5] K. Sasai, J. Sveholm, G. Kitagata and T. Kinoshita, "A Practical Design and Implementation of Active Information Resource based Network Management System”, vol. 2, no. 4, IJEIC, (2011), pp. 67-86.

[6] A. Kazi, D. M. Smith, K. Daniel, S. Zhong, P. Gupta and M. E. Bosley, "Potential Molecular Targets of Tea Polyphenols in Human Tumor Cells : Significance in Cancer Prevention”, In Vivo, (2002), pp. 397-403.

[7] S. C. Galloway and J. E. Graydon, "Uncertainty Symptom Distress and Information Needs After Surgery for Cancer of the Colon", Cancer Nursing, vol. 19, no. 2, (2006), pp. 112-117.

[8] C. S. Yang and X. Wang, "Green Tea and Cancer Prevention”, Nutr Cancer, vol. 62, (2010), pp. 931-937.

[9] C. F. Wu, C. T. Lin and P. M. Wang, "Applying Multi-Criteria Method to the Decision of Assessment Tools for High-care Student Groups”, vol. 6, no. 3, IJHIT, (2013), pp. 1-14.

[10] A. M. Davis, "A Strategy for Comparing Alternative Software Development Life Cycle Model”, IEEE Trans, Software Eng, (2008), pp. 1453-1461.

[11] W. G. Ouchi, "A Conceptual Framework for the Design of Organizational Control Mechanisms", Management Science, vol. 25, no. 9, (2009), pp. 833-848.

[12] K. J. Mayer and R. M. Salomon, "Capabilities, Contractual Hazards, and Governance, Integrating Resourcebased and Transaction Cost Perspectives", Academy of Management Journals, vol. 49, no. 6, (2006), pp. 942959.

[13] B. Tuttle, A. Harrell and P. Harrison, "Moral Hazard Ethical Considerations and the Decision to Implement an Information System", Journal of Management Information Systems, vol. 13, no. 4, (2007), pp. 7-25.

[14] L. Hao, “Application of MVC Platform in Bank E-CRM”, vol. 6, no. 2, IJUNESST, (2013), pp. 33-42.

[15] S. R. Lee, "Workshop on Healthcare and Nursing 2014 Third”, (2014); Jeju Island, Korea.

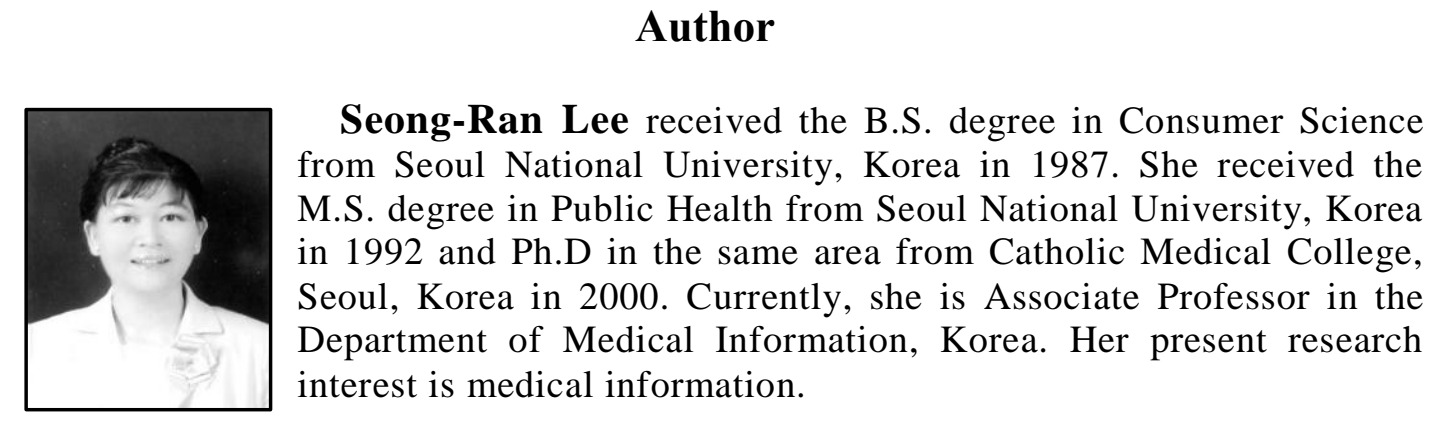


International Journal of Bio-Science and Bio-Technology Vol.6, No.5 (2014) 